\title{
ON SINGLE EQUATIONAL-AXIOM SYSTEMS FOR ABELIAN GROUPS
}

\author{
R. PADMANABHAN *
}

(Received 20 February 1967)

It is a fascinating problem in the axiomatics of any mathematical system to reduce the number of axioms, the number of variables used in each axiom, the length of the various identities, the number of concepts involved in the system etc. to a minimum. In other words, one is interested in finding systems which are apparently 'of different structures' but which represent the same reality. Sheffer's stroke operation and Byrne's brief formulations of Boolean algebras [1], Sholander's characterization of distributive lattices [7] and Sorkin's famous problem of characterizing lattices by means of two identities are all in the same spirit. In groups, when defined as usual, we demand a binary, unary and a nullary operation (respectively, say, $a, b \rightarrow a \cdot b ; a \rightarrow a^{-1}$; the existence of a unit element). However, as G. Rabinow first proved in [6], groups can be made as a subvariety of groupoids (mathematical systems with just one binary operation) with the operation $*$ where $a * b$ is the right division, $a b^{-1}$. In $[8]$, M. Sholander proved the striking result that a mathematical system closed under a binary operation $*$ and satisfying the identity $S$ : $x *((x * z) *(y * z))=y$ is an abelian group. Yet another identity, already known in the literature, characterizing abelian groups is $H N$ : $x *((z * y) *(z * x))=y$ which is due to G. Higman and B. H. Neumann $([3],[4]) *$. As can be seen both the identities are of length six and both of them belong to the same 'bracketting scheme' or 'bracket type'. In this paper we give four new identities of length six but of distinct bracket types $\left(\sum_{i}, i=1,2,3,4\right.$ of Theorem 1$)$ which characterize abelian groups individually and show that these six identities are the only identities of length six which can characterize abelian groups. Further we show that no identity of length less than six can have this property. Thus the above six identities are of minimal length. Since each one of the above identities contains just three variables they have the minimum number of variables in the sense of McKinsey and Diamond [2]. Thus $H N, S, \Sigma_{1}, \Sigma_{2}, \Sigma_{3}$ and $\Sigma_{4}$ are the simplest possible axiom systems for abelian groups in every sense of the word. They

* A University Grants Commission Research Fellow. 
are not of maximal length as the theoretical existence of an identity of length sixteen for abelian groups has been shown in [3]. In fact, to every positive integer $n$ one can make a law defining abelian groups (or, for that matter, groups), of a length exceeding $n$. Also one can make finite irredundant sets of laws, of arbitrarily large size, defining groups or abelian groups. So what we call 'simplest' is well-defined; but there is no opposite to it. For arbitrary groups this minimal length has been shown to be ten (see [5])

I wish to express my sincere thanks to Professor M. Venkataraman for his guidance and kind encouragement given to me throughout the prepation of this paper. My thanks are also due to Professor B. H. Neumann for his interest in this paper.

\section{Notation}

$G$ is a non-empty set and * is a binary operation in $G$ (to be interpreted as the analogue of the right division $a b^{-1}$ whenever $G$ is thought of a group). A word in $\langle G, *\rangle$ in which the free symbols $x_{1}, x_{2}, \cdots, x_{n}$ occur is denoted by $f\left(x_{1}, \cdots, x_{n}\right)$.

Definition. An identity is an equation of the form

$$
f\left(x_{1}, x_{2}, \cdots, x_{n}\right)=g\left(y_{1}, y_{2}, \cdots, y_{m}\right)
$$

which is supposed true for all $x^{\prime}$ 's and $y$ 's in $G$ (the sets $\left\{x_{1}, \cdots, x_{n}\right\}$ and $\left\{y_{1}, \cdots, y_{m}\right\}$ need not be disjoint - usually they will not be disjoint). If the word $f$ is of length $p$ and $g$ of length $q$ then the identity $f=g$ is said to be of length $p+q$ (For example, the associative law of group theory is an identity of length six).

Let $\langle G, *\rangle$ be a binary system satisfying a given set $\sum$ of identities. By the associated system we mean the mathematical system $\langle G, \cdot\rangle$ where we define $a \cdot b=a *((b * b) * b)$. We say $\langle G, *, \Sigma\rangle$ is an associated abelian group (an $a \cdot a \cdot$ group) if the associated binary system $\langle G, \cdot\rangle$ becomes an abelian group such that $a * b=a b^{-1}$. It is immediately verified that in an $a \cdot a \cdot$ group $\langle G, *, \Sigma\rangle$ the following identities must be true (i.e. deducible from $\Sigma$ ):

(4) $(x * z) *(y * z)=x * y$

(5) $(z * x) *(z * y)=y * x$.

1 This result is mentioned in [3]. 
For future reference we point out that the binary systems $\langle G, *,(3),(4)\rangle$ and $\langle G, *,(3),(5)\rangle$ are $a \cdot a \cdot$ groups. For, while by (3) and (4) we get $x *((x * z) *(y * z))=y$ which is the Sholander identity for abelian groups, (3) and (5) in a similar manner imply $H N$, the Higman-Neumann identity.

THeOREM 1. For a binary system $\langle G, *\rangle$ the following statements are equivalent:

$A:\langle G, *\rangle$ is an $a \cdot a \cdot$ group.

$\Sigma_{1}: x *(z *(y *(x * z)))=y$ for all $x, y, z$ in $G$.

$\Sigma_{2}: \quad(x *(z * y)) *(x * z)=y$ for all $x, y, z$ in $G$.

$\sum_{3}: \quad(x *((x * z) * y)) * z=y$ for all $x, y, z$ in $G$.

$\Sigma_{4}: \quad(x * z) *((x * y) * z)=y$ for all $x, y, z$ in $G$.

Proof. $A \Rightarrow \Sigma_{1}$ : It is immediately verified that in any $a \cdot a \cdot$ group identity $\sum_{1}$ holds.

$\Sigma_{1} \Rightarrow \Sigma_{2}$ : Replacing $z$ by $z *(y * x)$ in $\Sigma_{1}$, we get

$$
x *((z *(y * x)) *(y *(x *(z *(y * x)))))=y
$$

and this reduces, by $\Sigma_{1}$, to

$$
x *((z *(y * x)) * z)=y .
$$

Now replacing $x$ by $x *(z * y)$ and using $\sum_{1}$ again we get

which is $\sum_{2}$.

$$
(x *(z * y)) *(x * z)=y
$$

$\Sigma_{2} \Rightarrow \Sigma_{3}$ : First of all, let us observe that the system $\left\langle G, *, \Sigma_{2}\right\rangle$ is left-cancellative. For,

$a * b=a * c \Rightarrow(d *(a * b)) *(d * a)=(d *(a * c)) *(d * a) \Rightarrow b=c$ by $\Sigma_{\mathbf{2}}$.

Now, putting $x=z *(y * t)$ in $\Sigma_{2}$ and applying $\Sigma_{2}$ itself to the resulting identity we get

$$
t *((z *(y * t)) * z)=y .
$$

Thus we have, by (i) and the left-cancellation property,

$$
(z *(y * t)) * z=(x *(y * t)) * x .
$$

Putting $z=y *(t * s)$ in the above equation and applying $\Sigma_{2}$ we obtain

$$
s *(y *(t * s))=(x *(y * t)) * x .
$$

Putting $s=x *(y * t)$ in the above and applying the left-cancellation property we have

$$
y *(t *(x *(y * t)))=x
$$


Putting $t=r *(z *(y * r))$ in (i) and observing that $y * t=z$ by (ii) we have

$$
(r *(z *(y * r))) *((z * z) * z)=y .
$$

Now, replacing $r$ by $z * z$ and applying $\sum_{2}$ we get $y *(z * z)=y$, and hence

$$
x *(x * z)=(x *(z * z)) *(x * z)=z \text { by } \Sigma_{2} \text {. }
$$

Finally, replacing $z$ by $x * z$ in $\Sigma_{2}$ we get

$$
(x *((x * z) * y)) *(x *(x * z))=y
$$

and this reduces, by (iii), to

$$
(x *((x * z) * y)) * z=y
$$

which is the desired identity $\Sigma_{\mathbf{3}}$.

$\sum_{3} \Rightarrow \Sigma_{4}$ : Replacing $z$ by $(x * y) * t$ in $\Sigma_{3}$ and reducing the resulting identity by the given $\Sigma_{3}$ we get

$$
(x * t) *((x * y) * t)=y
$$

which is nothing but $\Sigma_{\mathbf{4}}$.

$\sum_{4} \Rightarrow A:$ Let $\sum_{4}:(x * z) *((x * y) * z)=y$ be valid in $\langle G, *\rangle$. Putting $z=x * y$ in $\Sigma_{4}$ we get $(x *(x * y)) *((x * y) *(x * y))=y$. Replacing $x$ by $x * y$ in the above equation and using $\sum_{4}$ we get

$$
y *(((x * y) * y) *((x * y) * y))=y \text {. }
$$

Calling $((x * y) * y) *((x * y) * y)=e(y, x)$ we have

$$
y * e(y, x)=y \text {. }
$$

Now putting $z=y=e(x, y)$ in $\Sigma_{4}$ and using (i) we find

$$
x * x=e(x, y) \text {. }
$$

Thus the element $e(x, y)$ is independent of its second argument $y$ and hence let us denote it simply by $e(x)$. Now (i) reads as $x * e(x)=x$. We have

$$
\begin{aligned}
x * x & =e(x, y) & & \\
& =((y * x) * x) *((y * x) * x) & & \\
& =((y * x) * x) *(((y * x) * e(y * x)) * x) & & \text { by (i) } \\
& =e(y * x) & & \text { by } \sum_{4} \\
& =(y * x) *(y * x) & & \text { by (ii) } \\
& =(y * x) *((y * e(y)) * x) & & \text { by (i) } \\
& =e(y) & & \text { by } \Sigma_{4} \\
& =y * y . & &
\end{aligned}
$$

Thus $x * x$ is a fixed element of $G$. Call this element $e$. Now (i) reads as $x * e=x$ for all $x$ in $G$. Putting $z=e$ in $\Sigma_{4}$ we find 


$$
x *(x * y)=y,
$$

and replacing $y$ by $x * y$ in $\Sigma_{4}$ and using (3) we obtain

$$
(x * z) *(y * z)=x * y \text {. }
$$

But as mentioned in the beginning of this section, the identities (3) and (4) will imply that the binary system $\langle G, *\rangle$ is an $a \cdot a \cdot$ group. Thus $\sum_{4} \Rightarrow A$ and this completes the proof of the theorem.

\section{2}

The identities given in the last section are of length six and we wish to assert that no other identity of length less than or equal to six can characterize an abelian group. To this end we will show the existence of some restrictions on axiom systems for $a \cdot a \cdot$ groups. All our axiom systems consist of identities.

Lemma 1. If $\left\langle G, *, \sum\right\rangle$ is an $a \cdot a \cdot$ group then $\sum$ must contain at least one identity of the form $f\left(x_{1}, x_{2}, \cdots, x_{n}\right)=x_{1}{ }^{2}$

Proof. Any identity in $\Sigma$ not of the form given above must either be of the form

$$
f\left(x_{2}, x_{3}, \cdots, x_{n}\right)=x_{1}
$$

(i.e. the symbol $x_{1}$ does not occur in the set of variables in the LHS) or of the form

$$
f\left(x_{1}, x_{2}, \cdots, x_{n}\right)=g\left(x_{1}, x_{2}, \cdots, x_{n}\right)
$$

(i.e. the symbol $*$ occurs in both sides of the identity). If an identity of the form ( $\alpha$ ) occurs in $\sum$ then giving some specified set of values to $x_{2}, x_{3}, \cdots, x_{n}$ and allowing $x_{1}$ to vary, we find that $G$ consists of just one element and thus $\sum$ is not an axiom system for arbitrary abelian groups. Thus, $\Sigma$ does not have an identity of the form given in the theorem means that every member of $\sum$ is of the form $(\beta)$. Now, consider the system $\langle G, *\rangle$ where $G$ has more than two elements and where

$$
a * b=0,0 \in G \text {, for all } a, b \text { in } G .
$$

Any identity of the form $(\beta)$ is satisfied in this system but $\langle G, *\rangle$ is not an $a \cdot a \cdot$ group, since for example, the identity $x *(x * y)=y$ fails to hold here. Thus, if $\langle G, *, \Sigma\rangle$ is an $a \cdot a \cdot$ group then $\sum$ must include an identity of the form $f\left(x_{1}, x_{2}, \cdots, x_{n}\right)=x_{1}$.

If a single identity $\sum$ in $\langle G, *\rangle$ can characterize the concept of the

2 This lemma is stated for asso-groups without proof in [3]. 
abelian group in the above sense then the following two things must happen, viz: (i) $\sum$ decoded in the -language must be a true sentence in the abelian group $\langle G, \cdot\rangle$ and (ii) it should be possible to deduce all abelian group theoretic axioms from $\sum$. Such an identity we call an abg-identity. So, by the Lemma 1, the $r h s$ of an abg-identity must be a single variable which also occurs in the expression on the lhs. If $f\left(x_{1}, x_{2}, \cdots, x_{n}\right)$ is any word in $\langle G, *\rangle$ its group theoretic form can be written in a unique way as

$$
x_{1}^{m_{1}} x_{2}^{m_{2}} \cdots x_{n}^{m_{n}}
$$

where $m_{1}, m_{2}, \cdots, m_{n}$ are integers. By the degree of the word $f$ we mean the integer $\sum_{n} m_{n}$. If $f$ is of even length $k$ then its degree (possibly $k-$ an even integer) is even. So no word of even length can be the lhs of an abgidentity by Lemma 1 . We will show some more restrictions for identities to be abg-identities which will ultimately yield the result that no word of length less than or equal to six (other than the lhs expressions of $H N, S$, $\Sigma_{1}, \Sigma_{2}, \Sigma_{3}$ and $\Sigma_{4}$ ) can be the lhs of an abg-identity.

LeMMA 2. In an abg-identity $\sum: f\left(x_{1}, x_{2}, \cdots, x_{n}\right)=x_{1}$, the element $x_{1}$ cannot occur either in the first place or in the last in the expression $f$.

Proof. Let the element $x_{1}$ occur as the first element in the lhs of $\sum$. Consider the system $\langle G, *\rangle$ where $a * b=a$ for all $a, b$ in $G$. $\sum$ is automatically satisfied in $G$ but the system is not an $a \cdot a \cdot$ group since, for example, the identity (3) fails to hold in the system. Similarly, by considering the algebra $G:\langle G, *\rangle$ where $a * b=b$ for all $a, b$ in $G$ we can prove the other result.

Lemma 3. In an abg-identity $\sum: f\left(x_{1}, x_{2}, \cdots, x_{n}\right)=x_{1}$ there exist at least two distinct variables $x_{i}$ other than $x_{1}$ (in the lhs) which do not occur only in the form $x_{i} * x_{i}$. In other words, if except two variables, every other variable $x_{i}$ occurs only in the form $x_{i} * x_{i}$ then $\sum$ cannot give rise to the axioms of abelian group.

Proof. Let $\sum: f\left(x_{1}, x_{2}, \cdots, x_{n}\right)=x_{1}$ be an abg-identity and let every variable other than $x_{1}$ and another variable, say $x_{2}$, occur only in the form $x_{i} * x_{i}$. We will arrive at a contradiction.

By what is given $\sum$ is of the form $g\left(x_{1}, x_{2}, x_{3} * x_{3}, \cdots, x_{n} * x_{n}\right)=x_{1}$. Since $\Sigma \Rightarrow$ (abelian) group axioms, the identity $x * x=y * y$ is a logical consequence of $\Sigma$. So we have $\Sigma \Rightarrow \Sigma^{\prime}$ where

$$
\Sigma^{\prime}=\left\{\begin{aligned}
\text { i } & g\left(x_{1}, x_{2}, x_{2} * x_{2}, \cdots, x_{2} * x_{2}\right)=x_{1} \\
\text { ii } & x * x=y * y
\end{aligned}\right\}
$$

Conversely, $\Sigma^{\prime} \Rightarrow \Sigma$ and hence the postulate system $\Sigma^{\prime}$ is logically equivalent to $\sum$. Thus the assumption that $\sum$ is an abg-identity leads to the conclusion that ' $\Sigma$ ' $\Rightarrow$ abelian group axioms' but $\Sigma$ ' is a set of identities 
such that each of its identity contains not more than two variables. This, however, is a contradiction due to a result of McKinsey and Diamond [2] according to which any axiom system for abelian groups must include at least one axiom which has three variables.

REMaRK. As observed in [5] these three lemmas hold good in any 'asso-group'. Since the main result in this section heavily depends on these results we have produced the proofs.

Before proceeding further we have to adapt some canonical way to express the various words of various lengths. The main difficulty arises out of the fact that the binary operation $*$ is not associative. To start with we have the simplest word $x_{1}$. Let us call this a word of type 1 . The most general word of length two is $x_{1} * x_{2}$. This family of words, viz. $x_{1} * x_{1}, x_{1} * x_{2}$, $x_{2} * x_{1}, x_{2} * x_{2}$ is denoted by bracket type 2 or simply by type 2 . The most general word of length three can be formed in two ways, that is $x_{1} *\left(x_{2} * x_{3}\right)$ and $\left(x_{1} * x_{2}\right) * x_{3}$. These families of words are respectively denoted by type $1+2$ and type $2+1$. Similarly there are five distinct types of length four and so on. The degree of words of type 1 is trivially 1 and that of type 2 is 0 . Suppose $f$ is of degree $m$ and $g$ is of degree $n$. Then the word $f * g=f g^{-1}$ has degree $m-n$. Thus all words belonging to a particular type have the same degree. Using these facts we calculate inductively the degrees of all words of length upto five. Among these we need only consider those words of degree one. Let $\beta$ be a type of degree one. If the free expression corresponding to $\beta$ can be reduced by some identifications, say

$$
x_{i_{0}}=x_{j_{0}}, \cdots, x_{i_{k}}=x_{j_{k}}, i_{r} \neq j_{r}, i_{r}, j_{r} \in\{1,2, \cdots, n\}
$$

so that it reduces to

$$
f\left(x_{1}, \cdots, x_{p}, \cdots, x_{n}\right)=x_{p}
$$

where $x_{p}$ is neither the first element nor the last element (i.e. neither occurs in the first place nor in the last place) in the expression $f$, then we say that the type $\beta$ has a non-trivial reduction. By Lemma 2, types which have only trivial reductions cannot yield abg-identities. In the Appendix below we list all types of words of length upto five, and give arguments to show that the resulting non-trivial reductions (other than the six known $a b g$-identities mentioned previously) cannot be abg-identities. The routine calculations involved in finding the degrees of the types etc. are left out. The following lemma will explain the general pattern of the proof given in the Appendix.

Lemma 4. No word belonging to the bracket type $1+((1+2)+1)$ can be the lhs of an abg-identity.

Proof. The form of the free expression corresponding to the given type is $x_{1} *\left(\left(x_{2} *\left(x_{3} * x_{4}\right)\right) * x_{5}\right)$. Since the group theoretic expansion of this 
expression is $x_{1} x_{5} x_{3} x_{4}^{-1} x_{2}^{-1}$ its only possible non-trivial reduction is $x_{3}$ and this can happen when and only when $x_{1} x_{5} x_{4}^{-1} x_{2}^{-1}=e$, the group identity. This equation can be effected in two ways, viz. $x_{1}=x_{2}, x_{4}=x_{5}$; or $x_{1}=x_{4}$, $x_{2}=x_{5}$. Thus the given type has only the following two identities as suitable candidates for an $a b g$-identity.

$$
\begin{aligned}
& S_{1}: x_{1} *\left(\left(x_{1} *\left(x_{3} * x_{4}\right)\right) * x_{4}\right)=x_{3} \\
& S_{2}: x_{1} *\left(\left(x_{2} *\left(x_{3} * x_{1}\right)\right) * x_{2}\right)=x_{3} .
\end{aligned}
$$

Let $G$ be the set $0,1,2,3,4,5,6,7$ and define $a * b=3(a-b)(\bmod 8)$ for all $a, b$ in $G$. We have

$$
\text { lhs of } \begin{aligned}
S_{1} & =x_{1} *\left(\left(x_{1} *\left(3 x_{3}-3 x_{4}\right)\right) * x_{4}\right) \\
& =x_{1} *\left(\left(3 x_{1}-9 x_{3}+9 x_{4}\right) * x_{4}\right) \\
& =x_{1} *\left(9 x_{1}-27 x_{3}+27 x_{4}-3 x_{4}\right) \\
& =3 x_{1}-27 x_{1}+81 x_{3} \\
& =x_{1}(\bmod 8), \text { the } r h s \text { of } S_{1} .
\end{aligned}
$$

Thus' the identity $S_{1}$ is satisfied in $\langle G, *\rangle$ but, since $x *(y * y)=3 x \neq x$ in general, $S_{1}$ is not an abg-identity.

Let us prove that the assumption that $S_{2}$ is an abg-identity leads to a contradiction. Indeed, if $S_{2}$ is an abg-identity then $S_{2} \Rightarrow S^{*}$ where

$$
S^{*}=\left\{\begin{array}{rrrr}
\text { i } & & (x * y) * x & =(x * x) * y \\
\text { ii } & & x * x & =y * y \\
\text { iij } & & (x * x) *(x * y) & =y * x \\
\text { iv } & & x *(x * y) & =y
\end{array}\right\}
$$

Since each one of the above sentences is true in an $a \cdot a \cdot$ group. Conversely, $S^{*} \Rightarrow S_{2}$, for,

$$
\begin{aligned}
x_{1} *\left(\left(x_{2} *\left(x_{3} * x_{1}\right)\right) * x_{2}\right) & =x_{1} *\left(\left(x_{2} * x_{2}\right) *\left(x_{3} * x_{1}\right)\right) & & \text { by (i) } \\
& =x_{1} *\left(\left(x_{3} * x_{3}\right) *\left(x_{3} * x_{1}\right)\right) & & \text { by (ii) } \\
& =x_{1} *\left(x_{1} * x_{3}\right) & & \text { by (iii) } \\
& =x_{3} & & \text { by (iv) } .
\end{aligned}
$$

Hence the axiom system $S^{*}$ is logically equivalent to $S_{2}$. Thus the assumption that $S_{2}$ is an abg-identity implies that the system $S^{*}$ is an axiom system for abelian groups but $S^{*}$ is a set of identities in which no identity contains more than two variables. As stated in Lemma 3 this is a contradiction due to the result of McKinsey and Diamond.

Conclusion. Thus the only identities of length less than or equal to seven which are abg-identities (i.e. which can characterize the concept of abelian groups individually) are $H N, S, \sum_{i}(i=1,2,3,4) .^{3}$ Since it is

s That the length five is minimum for abelian groups is mentioned in [3]. 
known that in the case of abelian groups $a * b=a b^{-1}$ and $a * b=a^{-1} b$ are the only binary operations which will make the class of abelian groups as a sub-variety of groupoids (see B. H. Neumann, Topics in Algebra (Universal algebra, 1962)) we have proved that these six identities are the only possible simplest axiom systems for abelian groups.

Lemmas 1,2 and 3 give certain necessary conditions for an identity in $\langle G, *\rangle$ to be an abg-identity. The problem of giving a characterization for abg-identities is open.

\section{APPENDIX}

\begin{tabular}{|c|c|c|c|c|}
\hline & Type & Degree & e Non-trivial reductions, if any & Whether an abg-identity or not \\
\hline 1 & 1 & 1 & - & - \\
\hline 2 & 2 & $\mathbf{0}$ & - & - \\
\hline 3 & $1+2$ & 1 & nil & - \\
\hline 3 & $2+1$ & -1 & - & - \\
\hline 4 & $1+(1+2)$ & $\mathbf{0}$ & - & - \\
\hline 4 & $1+(2+1)$ & 2 & - & - \\
\hline 4 & $2+2$ & 0 & - & - \\
\hline 4 & $(1+2)+1$ & $\mathbf{0}$ & - & - \\
\hline 4 & $(2+1)+1$ & -2 & - & - \\
\hline 5 & $1+(1+(1+2))$ & 1 & $\begin{array}{l}\left.x_{1} *\left(x_{1} *\left(x_{3} *\left(x_{1} * x_{4}\right)\right)\right)\right)=x_{3} \\
x_{1} *\left(x_{2} *\left(x_{3} *\left(x_{1} * x_{2}\right)\right)\right)=x_{3}\end{array}$ & $\begin{array}{l}\text { Ruled out by Lemma } 3 \\
\text { abg-identity; } \\
\sum_{1} \text { of Theorem } 1\end{array}$ \\
\hline 5 & $1+(1+(2+1))$ & -1 & - & - \\
\hline 5 & $1+(2+2)$ & 1 & $\begin{array}{l}x_{1} *\left(\left(x_{1} * x_{3}\right) *\left(x_{4} * x_{4}\right)\right)=x_{3} \\
x_{1} *\left(\left(x_{2} * x_{3}\right) *\left(x_{2} * x_{1}\right)\right)=x_{3} \\
x_{1} *\left(\left(x_{1} * x_{3}\right) *\left(x_{4} * x_{3}\right)\right)=x_{4} \\
x_{1} *\left(\left(x_{2} * x_{2}\right) *\left(x_{4} * x_{1}\right)\right)=x_{4}\end{array}$ & $\begin{array}{l}\text { Ruled out by Lemma } 3 \\
H N \text {, the Higman- } \\
\text { Neuman identity } \\
S \text {, the Sholander identity } \\
\text { Ruled out by Lemma } 3\end{array}$ \\
\hline 5 & $1+((1+2)+1)$ & $I$ & $\begin{array}{c}\text { Discussed completely in } \\
\text { the Lemma } 4\end{array}$ & $\begin{array}{c}- \\
-\end{array}$ \\
\hline 5 & $1+((2+1)+1)$ & 3 & - & - \\
\hline 5 & $2+(1+2)$ & -1 & - & - \\
\hline 5 & $\begin{array}{l}2+(2+1) \\
(1+2)+2\end{array}$ & 1 & $\begin{array}{l}\left(x_{1} * x_{1}\right) *\left(\left(x_{3} * x_{4}\right) * x_{3}\right)=x_{4} \\
\left(x_{1} * x_{2}\right) *\left(\left(x_{1} * x_{4}\right) * x_{2}\right)=x_{4} \\
\left(x_{1} *\left(x_{1} * x_{3}\right)\right) *\left(x_{4} * x_{4}\right)=x_{3} \\
\left(x_{1} *\left(x_{2} * x_{3}\right)\right) *\left(x_{1} * x_{2}\right)=x_{3}\end{array}$ & $\begin{array}{l}\text { Ruled out by Lemma } 3 \\
\sum_{4} \text { of Theorem } 1 \\
\text { Ruled out by Lemma } 3 \\
\Sigma_{2} \text { of Theorem } 1\end{array}$ \\
\hline 5 & $(2+1)+2$ & -1 & - & - \\
\hline 5 & $(1+(1+2))+1$ & -1 & - & - \\
\hline 5 & $(1+(2+1))+1$ & 1 & $\begin{array}{l}\left(x_{1} *\left(\left(x_{2} * x_{3}\right) * x_{2}\right)\right) * x_{1}=x_{3} \\
\left(x_{1} *\left(\left(x_{1} * x_{3}\right) * x_{4}\right)\right) * x_{3}=x_{4} \\
\left(x_{1} *\left(\left(x_{2} * x_{2}\right) * x_{4}\right)\right) * x_{1}=x_{4}\end{array}$ & $\begin{array}{l}\text { Not an } a b g \text {-identity: for, } \\
\text { in }\langle 0,1,2,3,4, *\rangle \\
\text { where } a * b=2 a-3 b(\bmod 5) \\
\text { this identity is true } \\
S^{*} \text { of Lemma } 4 \text { implies } \\
\text { this identity } \\
\sum_{3} \text { of Theorem } 1 \\
\text { Ruled out by Lemma } 3\end{array}$ \\
\hline 5 & $(2+2)+1$ & -1 & - & - \\
\hline 5 & $((1+2)+1)+1$ & -1 & - & - \\
\hline 5 & $((2+1)+1)+1$ & -3 & - & - \\
\hline
\end{tabular}


Added in Proof: The identity $\Sigma_{1}$ was found by A. Tarski in Funda. menta Mathematica 30 (1938). The author got this information through a prepublication copy of G. Grätzer's paper: 'On the spectra of classes of algebras', Proc. Amer. Math. Soc., vol. 18 (1967).

\section{References}

[1] L. Byrne, 'Two brief formulations of Boolean algebras', Bull. Amer. Math. Soc. 52 (1946), $269-272$.

[2] J. C. C. McKinsey and A. H. Diamond, 'Algebras and their subalgebras', Bull. Amer. Math. Soc. 53 (1947), $959-962$.

[3] G. Higman and B. H. Neuman, 'Groups as groupoids with one law', Publ. Math. Debrecen 2 (1952), 215-221.

While preparing this paper the author could not refer to the text of [3]. The only available reference was its review, i.e. [4].)

[4] G. Higman and B. H. Neumann, Review of [3], Mathematical Reviews 15 (1954), 284.

[5] R. Padmanabhan, 'On single equational-axiom systems for Groups' (to appear).

[6] G. Rabinow, 'Postulates for abelian groups', Amer. J. Math. 59 (1937), $211-224$.

[7] M. Sholander, 'Postulates for distributive lattices', Canad. J. Math. 3 (1951), 28-30.

[8] M. Sholander, 'Postulates for Commutative groups', Amer. Math. Monthly 66 (1959), 93 -95.

Department of Mathematics

Madurai University

Madurai 2 (India) 